\title{
Non-linear thermal resistance model for the simulation of high power GaN-based devices
}

\author{
S García-Sánchez ${ }^{1, *}$ (1) , I Íñiguez-de-la-Torre ${ }^{2}$ (D) S Pérez ${ }^{2}$ (i), K Ranjan $^{3}$, M Agrawal $^{3}$, \\ R Lingaparthi ${ }^{3}$, D Nethaji ${ }^{3}, K_{\text {Radhakrishnan }}^{3}$, S Arulkumaran ${ }^{3}$, G I Ng $^{3}$, T González ${ }^{2}$ \\ and $\mathrm{J}$ Mateos $^{2}$ (D) \\ ${ }^{1}$ Department of Physics and Mathematics, University of Alcalá de Henares, Madrid, Spain \\ 2 Department of Applied Physics, University of Salamanca, Salamanca, Spain \\ 3 Temasek Laboratories@ NTU, Nanyang Technological University, Singapore \\ E-mail: sergio.garcias@uah.es
}

Received 7 January 2021, revised 16 February 2021

Accepted for publication 3 March 2021

Published 29 March 2021

\begin{abstract}
We report on the modeling of self-heating in GaN-based devices. While a constant thermal resistance is able to account for the self-heating effects at low power, the decrease of the thermal conductance of semiconductors when the lattice temperature increases, makes necessary the use of temperature dependent thermal resistance models. Moreover, in order to correctly account for the steep increase of the thermal resistance of GaN devices at high temperature, where commonly used models fail, we propose a non-linear model which, included in an electro-thermal Monte Carlo simulator, is able to reproduce the strongly non-linear behavior of the thermal resistance observed in experiments at high DC power levels. The accuracy of the proposed non-linear thermal resistance model has been confirmed by means of the comparison with pulsed and DC measurements made in devices specifically fabricated on doped GaN, able to reach DC power levels above $150 \mathrm{~W} \mathrm{~mm}^{-1}$ at biases below $30 \mathrm{~V}$.
\end{abstract}

Keywords: electrothermal effects, GaN, power semiconductor devices, thermal resistance

(Some figures may appear in colour only in the online journal)

\section{Introduction}

When dealing with the design of high power devices, selfheating is one of the key aspects to take into account. Simple models using a constant thermal resistance, $R_{\mathrm{th}}$, can be applicable when the amount of dissipated power, $P_{\text {diss }}$, and the related temperature increase are not very high, so that their relationship can be correctly assumed to be linear, as in [1-4]. Deviations from this simple approximation were already experimentally observed in HBTs based on GaAs due to the temperature dependence of its thermal conductivity [5]. The use of a nonconstant $R_{\mathrm{th}}$, usually linearly dependent on the dissipated power $[6,7]$, on the lattice temperature, $T_{\text {latt }}[7,8]$,

\footnotetext{
Author to whom any correspondence should be addressed.
}

or both $[9,10]$, is widespread in electrothermal device models and commercial software like ANSYS $[11,12]$. However, the use of novel technologies based on wide bandgap semiconductors such as $\mathrm{GaN}, \mathrm{SiC}$ or even diamond, are strongly pushing the limits of the power density that can be managed by practical MMICs. In particular, GaN technology is becoming increasingly important for high-power RF applications [13] as the use of improved heterojunctions, with barrier materials like InGaN or AlN, is assisting in the increase of sheet electron density $n_{\mathrm{s}}$ to levels above $1.5 \times 10^{13} \mathrm{~cm}^{-2}$, while keeping a high electron mobility [14-16]. This allows reaching higher output currents and power densities, but consequently enhancing self-heating effects, which must be adequately managed to avoid the degradation of the devices and related reliability problems. Therefore, theoretical models precisely accounting for the self-heating of devices are of key importance. Improved 
thermal models accounting for the temperature dependence of the thermal conductivity of the semiconductors using a Kirchhoff transformation [17-20] are able to better reproduce the nonlinear self-heating effects appearing in devices based in such wideband gap semiconductors, but still are not enough to precisely describe their behaviour under ultra-high power bias conditions. As explained in [21], the double dependency of $R_{\text {th }}$ on $P_{\text {diss }}$ and $T_{\text {latt }}$ makes difficult to relate its values with the measured device temperature, mainly when $P_{\text {diss }}$ is very high.

In this paper, we tackle this problem from two different angles. On the one hand, we have fabricated specific highpower resistors based on a highly doped epilayer with $n_{\mathrm{s}}$ above $9.5 \times 10^{13} \mathrm{~cm}^{-2}$, thus providing very high $P_{\text {diss }}$, without applying too high bias, which somewhat simplifies the characterization tasks. On the other hand, we have used a selfconsistent electro-thermal Monte Carlo simulator to verify the lack of validity of commonly used (linear and non-linear) thermal resistance models at high-levels of $P_{\text {diss }}$ [20, 22-27]. Indeed, even if the decrease of the thermal conductivity with the temperature is well known [17-20, 26, 27], and is usually accounted for in commercial simulators like ANSYS [11, 12], the non-linear thermal resistance or full-electrothermal models, have only been verified to be valid in high electron mobility transistors (HEMTs) up to $P_{\text {diss }}$ around $10-20 \mathrm{~W} \mathrm{~mm}^{-1}$. Indeed, when $P_{\text {diss }}$ increases above those levels (or temperatures above $500 \mathrm{~K}$ are reached), the gate is degraded. That is why in this work we focus on resistors, which allow for a much larger $P_{\text {diss }}$ and thus make possible the validation of the models in a wider temperature range. In order to make the comparison, high-power ultra-short pulsed measurements were carried out by using an specific experimental setup to suppress almost completely the self-heating effects and verify the validity of our simulation model.

In this paper, we propose a novel non-linear thermal resistance model which allows reproducing the experimental $I-$ $V$ curve of the resistors up to extremely high values of $P_{\text {diss }}$ (above $150 \mathrm{~W} \mathrm{~mm}^{-1}$ ). However, the proposed model is general, and flexible enough so that it can also be applied to $\mathrm{AlGaN} / \mathrm{GaN}$ heterojunctions and HEMTs based on different substrates and material systems.

\section{Device details and Monte Carlo simulations}

A specific process for growing doped $\mathrm{GaN}$ on $4 \mathrm{H}$ semi insulating $\mathrm{SiC}$ substrate was developed and epilayers with the structure shown in the inset of figure 1 were obtained [28, 29]. The growth was started using AIN as a nucleation layer followed by the growth of a two-step $\mathrm{GaN}$ buffer layer to achieve smooth surface morphology and good crystalline quality. Finally, a $400 \mathrm{~nm}$ thick Si doped $n^{+}-\mathrm{GaN}$ active layer was grown. The Hall measurements made at different locations of a wafer grown under those conditions show mean values of $n=2.4 \times 10^{18} \mathrm{~cm}^{-3}, R_{\mathrm{s}}=360 \Omega$ and $\mu_{\mathrm{n}}=200 \mathrm{~cm}^{-2} \mathrm{~V}^{-1} \mathrm{~s}^{-1}$. At this point, it may be noted that even if the value of the square resistance of this epilayer is similar or even higher than that of $\mathrm{GaN}$ heterojunctions used for the fabrication of

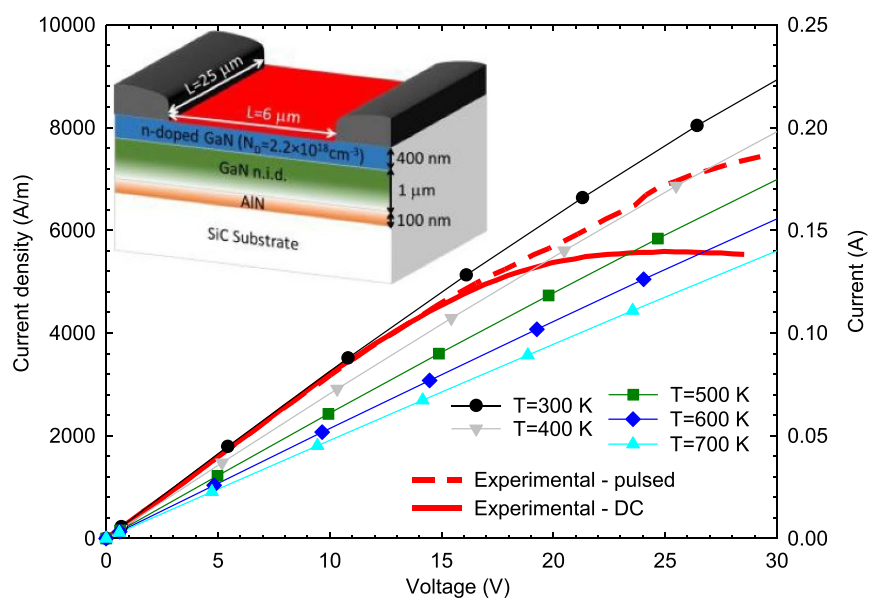

Figure 1. Comparison of the experimental $I-V$ curves of the structure (both in DC and with 300 ns pulsed measurements) with isothermal MC simulations (for different lattice temperatures from 300 to $700 \mathrm{~K})$.

HEMTs $[14,16]$, the corresponding $n_{\mathrm{s}}$ is much higher, about $9.6 \times 10^{13} \mathrm{~cm}^{-2}$ (almost ten times higher than the values typically found in HEMTs). This is due to a much lower value of the electron mobility in the bulk compared to that in the twodimensional electron gas, limited by the high doping level of our active layer. However, the saturation velocity is similar, so that the current level expected in our structure is almost ten times larger than that observed in HEMTs.

The details of the MC model used for the simulations of the diodes are given in [22-24]. In order to reproduce the mobility of the epilayers, a value $0.7 \times 10^{10} \mathrm{~cm}^{-2}$ was used for the dislocation density in the MC simulations, very near to the experimental one, of about $0.65 \times 10^{10} \mathrm{~cm}^{-2}$. It was estimated with high-resolution x-ray diffraction using the value of the FWHM of rocking curve scans along GaN (102). Ohmic contacts were fabricated to define resistors with different lengths and widths. TLM calibration structures provided values of the ohmic contact resistance $R_{\mathrm{C}}$ between 0.3 and $0.4 \Omega \mathrm{mm}$. The effect of the power dissipated at the contact resistances is added to our intrinsic MC simulations by evaluating (every certain number of iterations, $N_{\text {upd }}$ ) the extrinsic voltage as $V=V_{\mathrm{MC}}+2 R_{\mathrm{C}} I_{\mathrm{MC}}$, with $V_{\mathrm{MC}}$ being the intrinsic applied bias and $I_{\mathrm{MC}}$ the average current during that time interval. The DC extrinsic dissipated power is then calculated as $P_{\text {diss }}=I_{\mathrm{MC}} V$ and the device temperature, $T_{\text {latt }}$, is updated according to the thermal model of choice (and consequently the electron scattering probabilities) and used in the simulations during the following iterations until it is updated again. In fact, at every time moment the device is simulated at a uniform lattice temperature. For the electro-thermal simulations presented in this work, $T_{\text {latt }}$ is updated every $N_{\text {upd }}=6000$ iterations of 0.2 fs each.

\section{Thermal resistance models}

In the following we will consider different relationships between $T_{\text {latt }}$ and $P_{\text {diss }}$, based on the consideration of a thermal 
resistance, $R_{\mathrm{th}}[22,23]$, in what will be called thermal resistance models (TRMs).

\subsection{Constant $R_{\text {th }}(T R M-1)$}

The first approach is based on the use of a temperatureindependent thermal resistance, $R_{\mathrm{th}, a m b}$, so that $T_{\text {latt }}$ is updated according to the simple formula:

$$
T_{\text {latt }}=T_{\mathrm{amb}}+P_{\mathrm{diss}} R_{\mathrm{th}, \mathrm{amb}},
$$

meaning that the temperature increase in the device, $\Delta T$, provided by the Joule conversion of the electrical power into heat, is directly proportional to such power $P_{\text {diss }}$.

\subsection{Thermal-conductivity-related $R_{\text {th }}(T R M-2)$}

When the dissipated power is very high, a constant thermal resistance is not generally able to replicate the saturation nor the negative differential resistance of the $I-V$ experimental curve. In order to account for the steeper increase of the device temperature at high levels of $P_{\text {diss }}$, in this second model the value of $R_{\mathrm{th}}$ is not constant but follows the temperature dependence of the thermal conductivity. It is well know that thermal conductivity of semiconductors depends on the temperature according to, $k(T) \sim A T^{-\alpha}[20,25]$, where $A$ and $\alpha$ are characteristic parameters of each material [25]. As the thermal resistance is inversely proportional to the thermal conductivity, Paasschens et al [20] proposed a temperature dependent thermal resistance, $R_{\mathrm{th} 2}\left(T_{\text {latt }}\right)$, which will be used within this TRM-2:

$$
R_{\mathrm{th} 2}\left(T_{\text {latt }}\right)=R_{\mathrm{th}, \mathrm{amb}}\left(\frac{T_{\text {latt }}}{T_{\mathrm{amb}}}\right)^{\alpha_{\mathrm{eff}}}
$$

where $R_{\mathrm{th}, \mathrm{amb}}$, the thermal resistance at $T_{\mathrm{amb}}$, and $\alpha_{\text {eff }}$, its effective temperature-dependence exponent, are the parameters of the model. $\alpha_{\text {eff }}$ is usually taken as an adjustable parameter, since the device structure comprises different materials with different $k(T)$ dependencies. This is a strong assumption, which, as we will show later, hinders the validity of the model for large values of $P_{\text {diss }}$.

To calculate the increase of the real temperature $\Delta T$ in the device, the Kirchhoff variable transformation is used $[25,30,31]$ and the lattice temperature $T_{\text {latt }}$ is obtained as:

$$
T_{\text {latt }}=T_{\mathrm{amb}}\left[1+\frac{P_{\mathrm{diss}} R_{\mathrm{th}, \mathrm{amb}}\left(1-\alpha_{\mathrm{eff}}\right)}{T_{\mathrm{amb}}}\right]^{\frac{1}{1-\alpha_{e f f}}} .
$$

The lattice temperature in the device is therefore updated in the MC simulation according to the value of $P_{\text {diss }}$ in the corresponding time interval. Due to the non-linearity of [3], the increase in the device temperature $\Delta T=T_{\text {latt }}-T_{\mathrm{amb}}$ is not proportional to $P_{\text {diss }}$, and the temperature-dependent effective thermal resistance could be calculated as $R_{\mathrm{th} 2}^{\text {eff }}=\Delta T / P_{\text {diss }}$, which is different from the value of $R_{\mathrm{th}}$ given by [2]. For low $P_{\text {diss }}, R_{\text {th2 }}^{\text {eff }} \rightarrow R_{\text {th,amb. This model is also able to account for the }}$ commonly used approximation of a linear dependence of the thermal resistance with $P_{\text {diss }}$. Indeed, for low values of $P_{\text {diss }}$, equation (3) predicts a substantially linear variation of $R_{\mathrm{th} 2}^{\text {eff }}$ with both $T_{\text {latt }}$ and $P_{\text {diss }}$ [21] (leading to a second order dependence of $T_{\text {latt }}$ vs. $\left.P_{\text {diss }}\right)$.

\subsection{Ad-hoc temperature-dependent $R_{\text {th }}(T R M-3)$}

Since the TRM-2 is not able to correctly describe the steep increase in temperature at high levels of dissipated power, we propose an ad-hoc variant of the TRM-1 aiming to reproduce the experimental heating effects. Accordingly, we suggest introducing an extra term in [1] in order to account for the enhanced self-heating at high power levels as follows:

$$
T_{\text {latt }}=T_{\mathrm{amb}}+P_{\mathrm{diss}} R_{\mathrm{th}, \mathrm{amb}}\left(1+\left[\frac{P_{\mathrm{diss}} R_{\mathrm{th}, \mathrm{amb}}}{\Delta T_{0}}\right]^{\beta}\right),
$$

where $\beta$ (which has to be larger or equal to 1) and $\Delta T_{0}$, together with $R_{\mathrm{th}, \mathrm{amb}}$, are the parameters of the model (all depending, as the thermal resistance does, on the geometry of the devices and surrounding areas). The choice of this dependence, instead of other exponential or polynomial equations, was made in order to maintain the relationship between the model parameters and the physics of the self-heating effects, thus using an expression not much different to equation (3) from TRM-2. This allows to relate the value of the exponent $\beta$, as for TRM-2, to the temperature coefficient of the thermal conductivity of the different materials of the epilayer structure. The higher the temperature coefficients of the thermal conductivities of the semiconductors, $\alpha$ (and the possible presence of a thermal boundary resistance, TBR), the higher $\beta$. On the other hand, a linear dependence of $R_{\mathrm{th}}$ vs. $P_{\text {diss }}$ (as in $[6,7]$, and in commercial simulators like ANSYS software $[11,12]$ ) is obtained just by setting $\beta$ to 1 .

$\Delta T_{0}$ represents the value of $\Delta T$ from which a constant thermal resistance (linear dependence of $\Delta T$ vs. $P_{\text {diss }}$ ) is no longer able to describe the self-heating effects and the $\beta$ thpower term starts being relevant. If $\Delta T_{0}$ is set to a very large value, the linear behaviour of TRM-1 is recovered.

Again, the temperature-dependent effective thermal resistance could be calculated as $R_{\mathrm{th} 3}^{\mathrm{eff}}=\Delta T / P_{\mathrm{diss}}$, and, for low $P_{\text {diss }}$, $R_{\mathrm{th} 3}^{\mathrm{eff}} \rightarrow R_{\mathrm{th}, \mathrm{amb}}$.

The determination of the three parameters of the model ( $\Delta T_{0}, \beta$ and $R_{\mathrm{th}, a m b}$ ) is done as usual for the thermal models, by fitting the estimation of $T_{\text {latt }}$ with equation (4). First, the linear low power region is used to determine the value of $R_{\text {th,amb }}$. Then, $\Delta T_{0}$ is approximately obtained by observing the point when the $T_{\text {latt }}$ vs. $P_{\text {diss }}$ curve diverges from the linear dependence. Finally, $\beta>1$ is chosen so that the high-power non-linear behavior is correctly reproduced (it is a measure of the non-linearity of the $\Delta T$ vs. $P_{\text {diss }}$ dependence for values above $\Delta T_{0}$ ) and a fine-tuning of the parameters is made.

\section{Results and discussion}

In order to have a first picture of the influence of self-heating in the device characteristics, figure 1 shows the comparison between the experimental pulsed measurements of the $I-V$ 
curve of the device represented in the inset, with a length of $6 \mu \mathrm{m}$ and a width of $25 \mu \mathrm{m}$, and the isothermal simulations in the $300-700 \mathrm{~K}$ range. The $I-V$ curves were initially measured in DC. Later on, the pulsed $I-V$ characterization was conducted using an Accent DiVA D265 system, with an initial pulse width of $1 \mu$ s (and a duty cycle below $0.1 \%$ ), but the $I-V$ curve was the same as in DC. The pulse width was then decreased to $300 \mathrm{~ns}$, the minimum width allowed by our experimental setup. In this case, we observe that the current increases due to the suppressed self-heating, but, as it will be explained later, there is still some slight temperature increase at high voltages.

The simulated curve for $T=300 \mathrm{~K}$ shows a very good agreement with the measurements up to $10 \mathrm{~V}$, but above this bias heating effects start to be noticeable in the device. It is remarkable that, due to the very high electron concentration, the current level of the structure is above $7000 \mathrm{~A} \mathrm{~m}^{-1}$, with an absolute current reaching almost $0.18 \mathrm{~A}$, thus dissipating a massive DC power of about $4.5 \mathrm{~W}$ (or $180 \mathrm{~W} \mathrm{~mm}^{-1}$ ) for $25 \mathrm{~V}$ applied. Consequently, even for the $300 \mathrm{~ns}$ pulsed measurement, self-heating slightly above $400 \mathrm{~K}$ is observed for the highest applied biases, indicating an extremely fast temperature increase.

Concerning the DC measurements, self-heating decreases the current significantly, so that the measured current for high voltages corresponds to the values obtained from simulations at much higher temperatures (thus allowing us to obtain an estimation of the temperature of operation of the resistor). For example, the current value at around $19 \mathrm{~V}$ is well reproduced by the MC simulations at $400 \mathrm{~K}$, while those at 23.4 and $27.0 \mathrm{~V}$, correspond to the simulations at $500 \mathrm{~K}$ and $600 \mathrm{~K}$, respectively (and expected to reach $700 \mathrm{~K}$ at about $30 \mathrm{~V}$ ), thus evidencing a steeper increase of the temperature at higher levels of $P_{\text {diss }}$.

The previous analysis shows that isothermal simulations are not capable of reproducing the experimental $I-V$ curves, so that heating effects must be self-consistently incorporated in the simulations. Figure 2 shows the $I-V$ curves obtained from the electro-thermal simulations when the TRM-1 with different constant values for the thermal resistance, $R_{\mathrm{th}, \mathrm{amb}}$, is considered. The background colours represent the lattice temperature at each bias point for $R_{\mathrm{th}, \mathrm{amb}}=0.5 \mathrm{~K} \mathrm{~mm} \mathrm{~W}^{-1}$ according to [1]. The values used for $R_{\mathrm{th}, \mathrm{amb}}$ are in the range $0.3-2.0 \mathrm{~K} \mathrm{~mm} \mathrm{~W}^{-1}$, much below those typically found in $\mathrm{AlGaN} / \mathrm{GaN}$ epilayers on Si substrate $\left(14 \mathrm{~K} \mathrm{~mm} \mathrm{~W}^{-1}\right.$ in [23], around $20 \mathrm{~K} \mathrm{~mm} \mathrm{~W}^{-1}$ in [32] and $25 \mathrm{~K} \mathrm{~mm} \mathrm{~W}^{-1}$ in [33]), but also slightly lower than our previous theoretical estimations $\left(9 \mathrm{~K} \mathrm{~mm} \mathrm{~W}^{-1}\right.$ in [24]) and experimental values $\left(4 \mathrm{~K} \mathrm{~mm} \mathrm{~W}^{-1}\right.$ in [34] and $15 \mathrm{~K} \mathrm{~mm} \mathrm{~W}^{-1}$ in [35]) for the thermal resistance of heterostructures grown on $\mathrm{SiC}$. This is probably due to an improved value of the TBR [24], and the thinner buffer of our epilayer structure (1 $\mu \mathrm{m}$ as compared to $1.5 \mu \mathrm{m}$ in [24] and $2 \mu \mathrm{m}$ in [35]).

As seen in figure 2, the current density is not significantly affected by self-heating at low bias, up to $10 \mathrm{~V}$, even if the DC power is already significant (more than $30 \mathrm{~W} \mathrm{~mm}^{-1}$, or $0.75 \mathrm{~W}$ total power), due to the low values of the thermal resistance. Above that applied voltage, the higher the value of $R_{\mathrm{th}, \text { amb }}$ the stronger is the current saturation due to the enhanced thermal

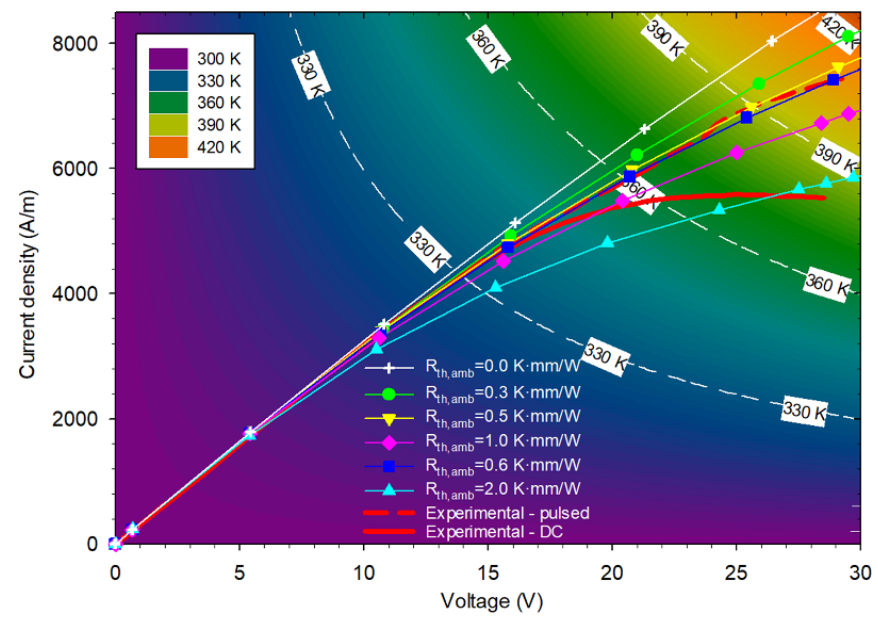

Figure 2. $I-V$ curves obtained with the TRM-1 for different values of the thermal resistance, $R_{\mathrm{th}, \mathrm{amb}}=0.3,0.5,0.6,1.0$ and

$2.0 \mathrm{~K} \mathrm{~mm} \mathrm{~W}^{-1}$, together with the isothermal simulation at $300 \mathrm{~K}$, compared with the measurements. The background colors represent the lattice temperature at each bias point for

$R_{\mathrm{th}, \mathrm{amb}}=0.5 \mathrm{~K} \mathrm{~mm} \mathrm{~W}^{-1}$.

effects. This simple model using a constant thermal resistance (TRM-1) is able to correctly reproduce the experimental measurements made with $300 \mathrm{~ns}$ pulses by using $R_{\mathrm{th}, \mathrm{amb}}$ around $0.5-0.6 \mathrm{~K} \mathrm{~mm} \mathrm{~W}^{-1}$. This is so because the thermal effects are limited in that kind of measurements, and the device temperature does not go much above $400 \mathrm{~K}$ (as seen in the color map of figure 2). On the other hand, the experimental DC current is only well reproduced up to around $16 \mathrm{~V}$. At higher bias, the saturation of the current is not well accounted for in these simulations, even when using higher values for $R_{\mathrm{th}, \mathrm{amb}}$, meaning that the self-heating at high power levels is underestimated by the TRM-1. At increasing $T_{\text {latt }}$ the thermal conductance of the semiconductors (and therefore the thermal resistance of the devices) decreases, and a temperature dependent model, such as TRM-2 or TRM-3 must be used.

Figure 3 shows the $I-V$ curves obtained with the TRM-2 using $R_{\mathrm{th}, \mathrm{amb}}=0.24$ and $0.6 \mathrm{~K} \mathrm{~mm} \mathrm{~W}^{-1}$ and $\alpha_{\mathrm{eff}}$ in the range $1.5-5$. By using $R_{\text {th,amb }}=0.6 \mathrm{~K} \mathrm{~mm} \mathrm{~W}^{-1}$ and $\alpha_{\text {eff }}$ around 4 , the DC $I-V$ curve is much better reproduced than with the TRM1. However, not only the value of $R_{\mathrm{th}, \mathrm{amb}}$ must be artificially increased, but the values used for $\alpha_{\text {eff }}$ need to be much above those used in the literature [36-40], which are in the range 1.32.0. This originates that in the $\sim 10-20 \mathrm{~V}$ span the current level is slightly lower than the experimental one, and the current decrease above $25 \mathrm{~V}$ is not well captured by the TRM-2, even for the highest values of $\alpha_{\text {eff }}$.

For a better understanding of the way the thermal models work, figure 4(a) shows $T_{\text {latt }}$ vs. $P_{\text {diss }}$ for TRM- 1 and TRM2 , obtained by means of [1] and [3], respectively. In addition, we have included an estimation of the real temperature of operation of our structures vs. $P_{\text {diss }}$, obtained by comparing the experimental current (under both DC and pulsed operation) with that obtained from the isothermal simulations. Figure 4(a) shows that the experimental $T_{\text {latt }}$ at low values of $P_{\text {diss }}$ is well reproduced using both TRM-1 and TRM-2 with 


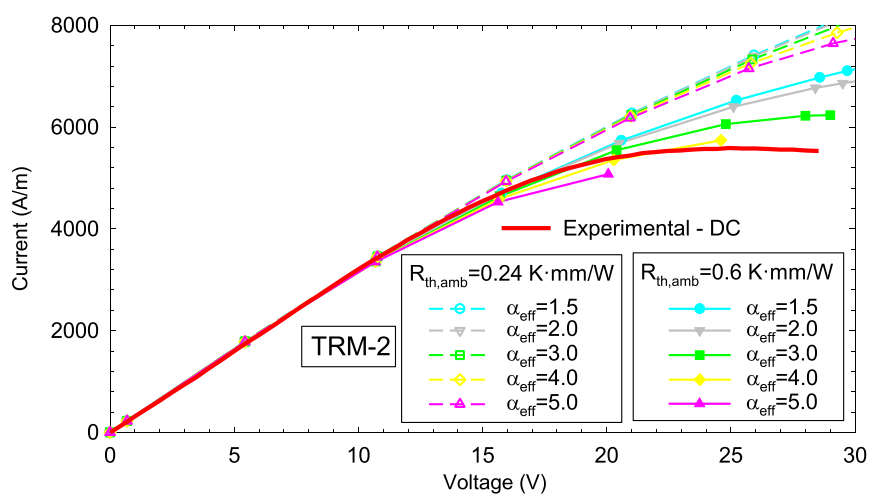

Figure 3. $I-V$ curves obtained with the TRM-2 for $R_{\text {th,amb }}=0.24$ and $0.6 \mathrm{~K} \mathrm{~mm} \mathrm{~W}^{-1}$, and $\alpha_{\text {eff }}$ from 1.5 to 5.0, compared with the DC measurements.

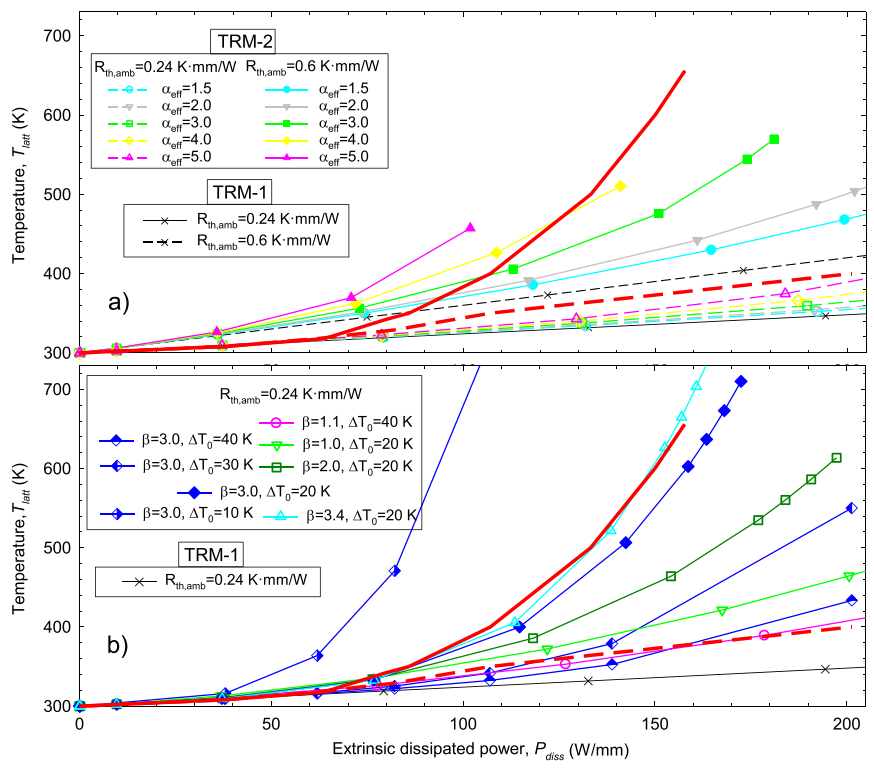

Figure 4. Lattice temperature vs. dissipated power obtained with (a) TRM-1 and TRM-2 with the parameters used in figures 2 and 3 and (b) TRM-1 and TRM-3 using $R_{\mathrm{th}, \mathrm{amb}}=0.24 \mathrm{~K} \mathrm{~mm} \mathrm{~W}^{-1}$, compared with the temperatures estimated from the experimental DC (solid) and pulsed (dashed) measurements.

$R_{\mathrm{th}, \mathrm{amb}}=0.24 \mathrm{~K} \mathrm{~mm} \mathrm{~W}^{-1}$. The more pronounced slope at high levels of $P_{\text {diss }}$ can only be approached by using TRM2 and much increasing $\alpha_{\text {eff }}$. However, its value cannot be too high because when the factor $P_{\mathrm{diss}} R_{\mathrm{th}, \mathrm{amb}}$ increases, the quantity $\left(1+P_{\text {diss }} R_{\text {th,amb }}\left(1-\alpha_{\text {eff }}\right) / T_{\text {amb }}\right)$ takes negative values and TRM- 2 cannot be used anymore, since $T_{\text {latt }}$ cannot be computed by means of [3]. Therefore, in order to come close to the experimental results, $R_{\mathrm{th}, \mathrm{amb}}$ has to be increased to $0.6 \mathrm{~K} \mathrm{~mm} \mathrm{~W}^{-1}$, but in this case the temperature increase is overestimated in the range of moderate $P_{\text {diss }}(\sim 50$ $100 \mathrm{~W} \mathrm{~mm}^{-1}$ ), while it is underestimated for the highest biases (even when using unrealistically high values of $\alpha_{\text {eff }}$ ).

We have to remark here that the TRM-2 is linked to the temperature dependence of the thermal conductivity of the various semiconductors present in the layer structure of the resistor. However, each one of them may have strongly different

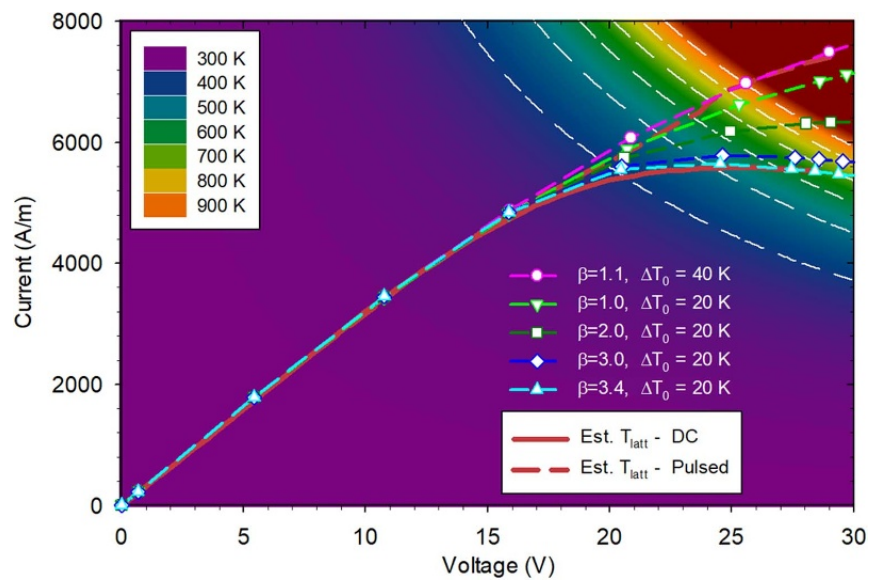

Figure 5. $I-V$ curves obtained with the TRM-3 using $R_{\mathrm{th} \text {,amb }}=0.24 \mathrm{~K} \mathrm{~mm} \mathrm{~W}^{-1}$ for different values of $\boldsymbol{\beta}$ and $\Delta \boldsymbol{T}_{0}$. The $\mathrm{DC}$ and pulsed experimental measurements are also plotted. The background colors represent the lattice temperature for each extrinsic bias point for the TRM-3 with $\boldsymbol{\beta}=3.4, \Delta \boldsymbol{T}_{0}=20 \mathrm{~K}$.

dependencies, therefore, TRM-2 needs to make use an effective parameter $\alpha_{\text {eff }}$, which does not seem to correctly reproduce the global thermal behaviour of the device. Conversely, in our structure the thermal conductivities of $\mathrm{GaN}$ and $\mathrm{SiC}$ have similar dependencies with $T_{\text {latt }}(\alpha=1.4$ for $\mathrm{GaN}$ and 1.3 for $\mathrm{SiC}$ [36]). Therefore, the problems may be related to the presence of the TBR of the $\mathrm{GaN} / \mathrm{SiC}$ transition, mainly determined by the low thermal conductivity within the AlN nucleation layer and the GaN interfacial region. The temperature dependence of TBR on $T_{\text {latt }}$ is completely different from those of the thermal conductivities of the semiconductors and depends a lot on the growing technology [41]. Indeed, in [36] the TBR of the GaN/SiC interface was estimated to have a temperature coefficient of $\alpha=2.76$, much different from those of $\mathrm{GaN}$ and $\mathrm{SiC}$. Due to these different dependencies, the contribution of the TBR to the self-heating of the device seems to become more important as $T_{\text {latt }}$ increases.

Finally, to overcome the drawbacks of the TRM-2 and try to adequately reproduce the temperature increase in our samples, we have implemented the ad-hoc TRM-3. It is noteworthy here that due to the strong dependence of $T_{\text {latt }}$ vs. $P_{\text {diss }}$ at high levels of $P_{\text {diss }}$, the intrinsic time-domain stochastic nature of MC simulations leads to spurious current oscillations. In order to solve this issue, instead of instantaneously updating the value of $T_{\text {latt }}$, we have introduced a softening parameter in these simulations that slows down the cooling of the device and is able to dampen this numerical instability. Figure 4(b) shows how the $T_{\text {latt }}$ vs. $P_{\text {diss }}$ dependence is affected by the parameters of the model, $\beta$ and $\Delta T_{0}$ (with $R_{\text {th,amb }}=0.24 \mathrm{~K} \mathrm{~mm} \mathrm{~W}^{-1}$ ): the higher is $\beta$ the sharper is the increase of $T_{\text {latt }}$, and takes place at lower levels of $P_{\text {diss }}$ the smaller is $\Delta T_{0}$. By setting the values of the parameters to $\beta=3.4, \Delta T_{0}=20 \mathrm{~K}$ and $R_{\mathrm{th}, \mathrm{amb}}=0.24 \mathrm{~K} \mathrm{~mm} \mathrm{~W}^{-1}$, the TRM-3 well reproduces the $T_{\text {latt }}$ vs. $P_{\text {diss }}$ curve, and hence the experimental $I-V$ curve, as shown in figure 5. Remarkably, the saturation of the current and even its slight decrease is well captured by TRM-3. Moreover, the results of the pulsed measurements can also be well reproduced by this model by setting 
$\beta=1.1$ and $\Delta T_{0}=40 \mathrm{~K}$ (providing approximately almost constant values for $R_{\mathrm{th}}^{\mathrm{eff}}$ ). However, this case is not much significant, because when pulses of $300 \mathrm{~ns}$ are applied we measure an instantaneous picture of a transient situation, in which $T_{\text {latt }}$ is not constant with time.

We have to remark that this is a general and flexible model for self-heating effects that can be applied to any kind of device or material system. In our previous work [24] we have demonstrated, by comparing the MC results using a simple thermal resistance model with those obtained with the detailed resolution of the heat flux equation (and providing the real temperature distribution inside the device), that the non-uniformity of the temperature in AlGaN/GaN HEMTs is not much important for simulating the correct electrical characteristics of the transistor. The temperature difference between the drain side of the gate (where the hot-point is located) and the rest of the channel is not very high, so that a thermal resistance model able to reproduce the average temperature of the channel is enough to provide correct electrical results. Therefore, the model developed in this paper can be of direct application also to high power HEMTs. Moreover, since the specific temperature coefficients of the thermal conductivity of the different materials (or even packaging) can be globally accounted for in the model by an appropriate fitting of the parameters, the proposed TRM-3 can also be applied to devices fabricated on other wide bandgap material systems like $\mathrm{SiC}$ or diamond.

\section{Conclusions}

We have fabricated resistors based on highly doped GaN epilayers allowing us to achieve extremely high DC power levels (above $150 \mathrm{~W} \mathrm{~mm}^{-1}$ ) at relative low applied voltages (below $30 \mathrm{~V}$ ), thus being strongly affected by self-heating effects. The devices have been characterized by means of a setup for fast-pulsed high-power electrical measurements. We have used them together with self-consistent Monte Carlo electro-thermal simulations to precisely calibrate the operation temperature of the GaN devices, analyze the (not completely satisfactory) results afforded by existing models and use an ad-hoc non-linear thermal resistance model able to correctly reproduce the thermal effects observed in the experiments even at very high levels of dissipated power.

\section{Acknowledgments}

This work was partially supported by the NRF2017-NRFANR003 GaNGUN project, the Spanish MINECO and FEDER through project TEC2017-83910-R and the Junta de Castilla y León and FEDER through project SA254P18.

\section{ORCID iDs}

S García-Sánchez (D) https://orcid.org/0000-0001-8061-4835
I Íñiguez-de-la-Torre (D) https://orcid.org/0000-0002-2059-

0955

S Pérez (D) https://orcid.org/0000-0002-1494-0795

J Mateos (D) https://orcid.org/0000-0003-4041-7145

\section{References}

[1] Dawson D E, Gupta A K and Salib M L 1992 CW measurement of HBT thermal resistance IEEE Trans. Electron Devices $392235-9$

[2] Zweidinger D T, Fox R M, Brodsky J S, Jung T and Lee S 1996 Thermal impedance extraction for bipolar transistors IEEE Trans. Electron Devices 43 342-6

[3] Vanhoucke T, Boots H and Van Noort W 2004 Revised method for extraction of the thermal resistance applied to bulk and SOI SiGe HBTs IEEE Electron Device Lett. 25 150-2

[4] Kuball M and Pomeroy J W 2016 A review of Raman thermography for electronic and opto-electronic device measurement with submicron spatial and nanosecond temporal resolution IEEE Trans. Device Mater. Reliab. 16 667-84

[5] Liu W and Yuksel A 1995 Measurement of junction temperature of an $\mathrm{AlGaAs} / \mathrm{GaAs}$ heterojunction bipolar transistor operating at large power densities IEEE Trans. Electron Devices 42 358-60

[6] Sewell J et al 1996 Thermal characterization of thermally-shunted heterojunction bipolar transistors IEEE Electron Device Lett. 17 19-21

[7] Menozzi R, Barrett J and Ersland P 2005 A new method to extract HBT thermal resistance and its temperature and power dependence IEEE Trans. Device Mater. Reliab. 5 595-601

[8] Grossman P C, Gutierrez-Aitken A, Kaneshiro E, Sawdai D and Sato K 2002 Characterization and measurement of non-linear temperature rise and thermal resistance in InP heterojunction bipolar transistors. Conf. Proc. 14th Indium Phosphide and Related Materials Conf. (Piscataway, NJ: IEEE) pp 83-6

[9] Bovolon N, Baureis P, Muller J, Zwicknagl P, Schultheis R and Zanoni E 1998 A simple method for the thermal resistance measurement of AlGaAs/GaAs heterojunction bipolar transistors IEEE Trans. Electron Devices 45 1846-8

[10] Santarelli A, Di Giacomo V, Cignani R, D’Angelo S, Niessen D and Filicori F 2010 Nonlinear thermal resistance characterization for compact electrothermal GaN HEMT modelling The 5th European Microwave Integrated Circuits Conf. (Piscataway, NJ: IEEE) pp 82-5

[11] Zhao X, Xu R and Xu Y 2015 An improved nonlinear thermal resistance extraction method for AlGaN/GaN HEMTs 2015 IEEE Int. Conf. on Communication Problem-Solving (ICCP) (Piscataway, NJ: IEEE) pp 261-3

[12] Pomeroy J W, Uren M J, Lambert B and Kuball M 2015 Operating channel temperature in GaN HEMTs: DC versus RF accelerated life testing Microelectron. Reliab. 55 2505-10

[13] Roccaforte F and Leszczynski M 2020 Nitride Semiconductor Technology: Power Electronics and Optoelectronic Devices (New York: Wiley)

[14] Medjdoub F, Carlin J, Gaquiere C, Grandjean N and Kohn E 2008 Status of the emerging InAlN/GaN power HEMT technology Open Electr. Electron. Eng. J. 21

[15] Medjdoub F, Zegaoui M, Waldhoff N, Grimbert B, Rolland N and Above R P $2011600 \mathrm{mS} / \mathrm{mm}$ transconductance with $2.3 \mathrm{~A} / \mathrm{mm}$ drain current density AlN/GaN 
high-electron-mobility transistors grown on silicon Appl. Phys. Express 4064106

[16] Hickman A, Chaudhuri R, Bader S J, Nomoto K, Lee K, Xing H G and Jena D 2019 High breakdown voltage in RF AlN/GaN/AIN quantum well HEMTs IEEE Electron Device Lett. 40 1293-6

[17] Yeats B 1992 GaAs reliability workshop Paper IV-1

[18] Liou L L, Ebel J and Huang C 1993 Thermal effects on the characteristics of $\mathrm{AlGaAs} / \mathrm{GaAs}$ heterojunction bipolar transistors using two-dimensional numerical simulation IEEE Trans. Electron Devices 40 35-43

[19] Yeats B 1999 Inclusion of topside metal heat spreading in the determination of HBT temperatures by electrical and geometrical methods [GaAs devices] GaAs IC Symp. IEEE Gallium Arsenide Integrated Circuit Symp. 21st Annual Technical Digest 1999 (Piscataway, NJ: IEEE) pp 59-62

[20] Paasschens J C J, Harmsma S and Van Der Toorn R 2004 Dependence of thermal resistance on ambient and actual temperature Bipolar/BiCMOS Circuits and Technology, 2004 Proc. 2004 Meeting (Piscataway, NJ: IEEE) pp 96-9

[21] Scott J 2001 Reconciliation of methods for bipolar transistor thermal resistance extraction. ISCAS 2001. The 2001 IEEE Int. Symp. on Circuits and Systems (Piscataway, NJ: IEEE) pp 465-8

[22] Mateos J, Perez S, Pardo D and Gonzalez T 2009 Monte Carlo analysis of thermal effects in GaN HEMTs 2009 Spanish Conf. on Electron Devices (Piscataway, NJ: IEEE) pp $459-62$

[23] García S, Iñiguez-de-la-torre I, García-Pérez O, Mateos J, González T, Sangaré P, Gaquière C and Pérez S 2015 Self-consistent electro-thermal simulations of $\mathrm{AlGaN} / \mathrm{GaN}$ diodes by means of Monte Carlo method Semicond. Sci. Technol. 30035001

[24] García S, Íñiguez-de-la-torre I, Mateos J, González T and Pérez S 2016 Impact of substrate and thermal boundary resistance on the performance of AlGaN/GaN HEMTs analyzed by means of electro-thermal Monte Carlo simulations Semicond. Sci. Technol. 31065005

[25] Bonani F and Ghione G 1995 On the application of the Kirchhoff transformation to the steady-state thermal analysis of semiconductor devices with temperature-dependent and piecewise inhomogeneous thermal conductivity Solid State Electron. 38 1409-12

[26] Darwish A, Bayba A J and Hung H A 2015 Channel temperature analysis of GaN HEMTs with nonlinear thermal conductivity IEEE Trans. Electron Devices $62840-6$

[27] Wang C, Xu Y, Yu X, Ren C, Wang Z, Lu H, Chen T, Zhang B and Xu R 2014 An electrothermal model for empirical large-signal modeling of $\mathrm{AlGaN} / \mathrm{GaN}$ HEMTs including self-heating and ambient temperature effects IEEE Trans. Microw. Theory Tech. 62 2878-87

[28] Agrawal M et al $2019 \mathrm{GaN}-$ based SSD structure for THz applications 2019 IEEE Asia-Pacific Microwave Conf. (APMC) (Piscataway, NJ: IEEE) pp 213-5
[29] Mateos J et al 2019 Design and fabrication of planar gunn nanodiodes based on doped GaN 2019 IEEE Asia-Pacific Microwave Conf. (APMC) (Piscataway, NJ: IEEE) pp 971-3

[30] Sadi T, Kelsall R W and Pilgrim N J 2006 Investigation of self-heating effects in submicrometer GaN/AlGaN HEMTs using an electrothermal Monte Carlo method IEEE Trans. Electron Devices 53 2892-900

[31] Batty W, Christoffersen C E, Panks A J, David S, Snowden C M and Steer M B 2001 Electrothermal CAD of power devices and circuits with fully physical time-dependent compact thermal modeling of complex nonlinear 3-d systems IEEE Trans. Compon. Packag. Manuf. Technol. 24 566-90

[32] Conway A, Asbeck P, Moon J and Micovic M 2008 Accurate thermal analysis of GaN HFETs Solid State Electron. 52 637-43

[33] Choi S, Heller E R, Dorsey D, Vetury R and Graham S 2012 The impact of bias conditions on self-heating in AlGaN/GaN HEMTs IEEE Trans. Electron Devices 60 159-62

[34] Pavlidis G, Hilton A, Brown J, Heller E and Graham S 2020 Monitoring the Joule heating profile of $\mathrm{GaN} / \mathrm{SiC}$ high electron mobility transistors via cross-sectional thermal imaging J. Appl. Phys. 128075705

[35] Pomeroy J W, Bernardoni M, Dumka D, Fanning D and Kuball M 2014 Low thermal resistance GaN-on-diamond transistors characterized by three-dimensional Raman thermography mapping Appl. Phys. Lett. 104083513

[36] Sarua A, Ji H, Hilton K P, Wallis D J, Uren M J, Martin T and Kuball M 2007 Thermal boundary resistance between GaN and substrate in $\mathrm{AlGaN} / \mathrm{GaN}$ electronic devices IEEE Trans. Electron Devices 54 3152-8

[37] Vitanov S, Palankovski V, Maroldt S and Quay R 2010 High-temperature modeling of AlGaN/GaN HEMTs Solid State Electron. 54 1105-12

[38] Sodan V, Stoffels S, Oprins H, Decoutere S, Altmann F, Baelmans M and De Wolf I 2018 Fast and distributed thermal model for thermal modeling of GaN power devices IEEE Trans. Compon. Packag. Manuf. Technol. 8 1747-55

[39] Mion C, Muth J, Preble E and Hanser D 2006 Accurate dependence of gallium nitride thermal conductivity on dislocation density Appl. Phys. Lett. 89092123

[40] Morelli D, Heremans J and Slack G 2002 Estimation of the isotope effect on the lattice thermal conductivity of group IV and group III-V semiconductors Phys. Rev. B 66195304

[41] Manoi A, Pomeroy J W, Killat N and Kuball M 2010 Benchmarking of thermal boundary resistance in $\mathrm{AlGaN} / \mathrm{GaN}$ HEMTs on SiC substrates: implications of the nucleation layer microstructure IEEE Electron Device Lett. 31 1395-7 